\title{
Surface structure changes of pathological placenta tissue observed using scanning electron microscopy - a pilot study
}

\author{
Rafał Bobiński ${ }^{1}$ Anna Pielesz², Wioletta Waksmańska1凶, Ewa Sarna², \\ Izabela Ulman-Włodarz1,3, Justyna Kania³, Monika Mikulska' and Agnieszka Turbiarz
}

'Faculty of Health Sciences, Department of Nursing and Emergency Medicine, University of Bielsko-Biala, Bielsko-Biala, Poland; ${ }^{2}$ Faculty of Materials, Civil and Environmental, Engineering, University of Bielsko-Biala, Bielsko-Biala, Poland; ${ }^{3}$ Department Gyneacology, Provincial Specialist Hospital, Tychy, Poland

Structural changes within the placenta are observed in the course of pathological pregnancy. The aim of the study was to perform initial assessment of morphological features of placenta. The analysis was conducted by Scanning Electron Microscopy. Samples of placenta of women who delivered neonates appropriate for gestational age were characterized by a homogenous surface texture with natural corrugation. The surface of IUGR placenta from the group of mothers with pregnancy induced hypertension was definitely heterogeneous - noticeable swelling of tissue surface was observed. Samples from LGA group also demonstrated a number of surface bulges and heterogeneities which were, nonetheless, characterized by a certain repeatability.

Key words: hypertension, pathological pregnancy, placenta

Received: 18 February, 2017; revised: 28 March, 2017; accepted: 28 March, 2017; available on-line: 12 August, 2017

e-mail: wwaksmanska@ath.bielsko.pl

Abbreviations: AGA, appropriate for gestational age; IUGR, intrauterine growth restriction; LGA, large for gestational age; SEM, scanning electron microscopy

\section{INTRODUCTION}

Maternal-fetal exchange of nutrients and metabolites occurs across the placental barrier. This barrier is a multilayered structure which separates maternal and fetal circulations (Elad et al., 2014). All substances have to pass through the villous trophoblast which consists of two membranes: the microvillus facing the maternal bloodstream and the basal facing the fetal bloodstream (Duttaroy, 2009; Haggarty, 2002; Haggarty, 2004). Transportation of the above mentioned substances across the placental barrier involves numerous passive and active mechanisms. Both microvillus and basal membranes of the syncytiotrophoblast demonstrate a complex structure with a number of surface-anchored macromolecules including receptors, enzymes and other important substances (Bobiński \& Mikulska, 2015). Proper functioning of this complex system is ensured by adequate intrauterine nutrition, i.e. providing the developing child with the right amount of building and energetic components as well as many other micro- and macromolecules (Bobiński et al., 2013a; 2013b). In the course of numerous diseases in both mother and fetus an alteration of trophoblast structures responsible for the transport of nutrients and metabolites may occur, which in turn impairs fetal development. An alteration of placenta in the course of pathology causes morphological changes visible via transmission and scanning electron microscope and changes in the immunohistochemical image (Atwaa \& Farghalyb, 2011; Selim et al., 2013). This article presents the results of pilot studies of surface of placentas membrane performed by scanning electron microscopy (SEM).

The aim of the study was to preliminarily assess the morphological features of the surface of placentas selected out of four groups: healthy women who delivered neonates appropriate for gestational age (AGA), healthy mothers who delivered neonates with intrauterine growth restriction (IUGR), mothers with pregnancy-induced hypertension who delivered neonates with IUGR, healthy mothers who delivered large-for-gestational-age neonates (LGA).

\section{MATERIAL AND METHODS}

Study population. The study population consisted of 16 women who gave birth at the Provincial Specialist Hospital No. 1 in Tychy, Poland. Pregnant women were recruited for the study during their first visit to the hospital. The women were between 21 and 44 years of age.

To obtain a homogeneous group of women, the following inclusion criteria were applied:

- Polish nationality (excluding naturalized Polish citizens); single pregnancy; pregnancy I-III (parity considered);

- stable socioeconomic status; married, secondary level or higher education; living in a highly industrialized urban region, both the women and their husbands having a steady job.

- granting consent to participate in the study.

The following exclusion criteria were applied:

- chronic diseases occurring in the women before pregnancy, such as pre-gestational diabetes; pathologies during the course of pregnancy such as infections during pregnancy (any kind of infection in the perinatal period, such as fever, respiratory infections, urinary infections, etc.); miscarriages and/or premature birth resulting in the death of the child or developmental anomalies in the fetus;

- AIDS and sexually transmitted diseases;

- adherence to a vegetarian diet, mediterranean diet, or any other special diet;

- lack of the mother's consent to take part in the research program or withdrawal of consent during the study.

Women who participated in the research program were divided into four groups according to the following criteria: 


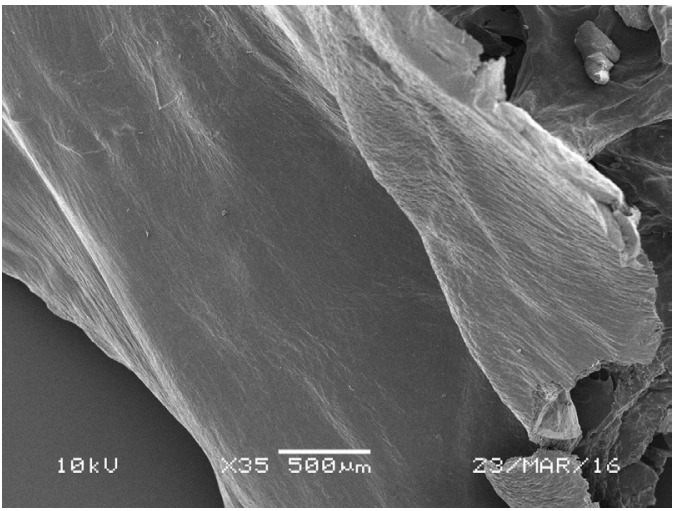

B

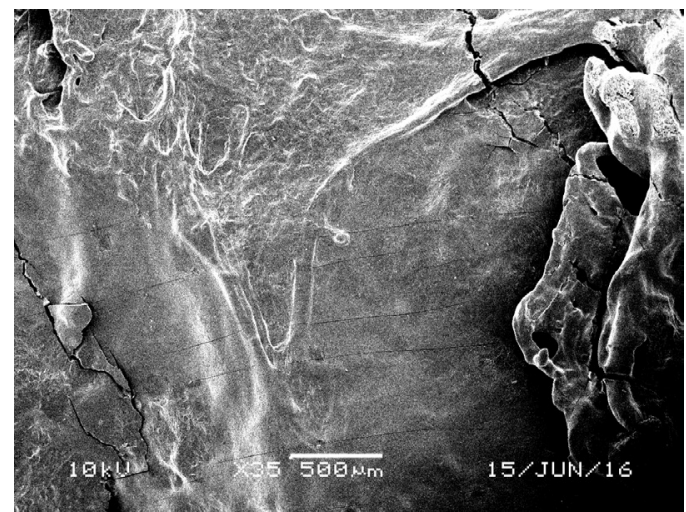

C

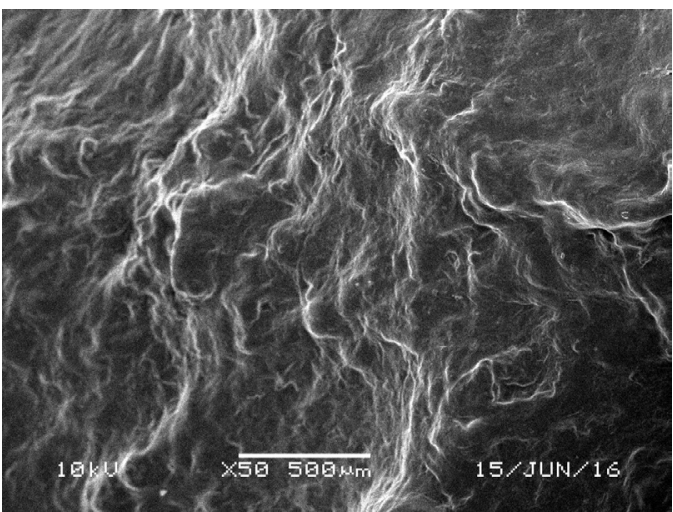

D

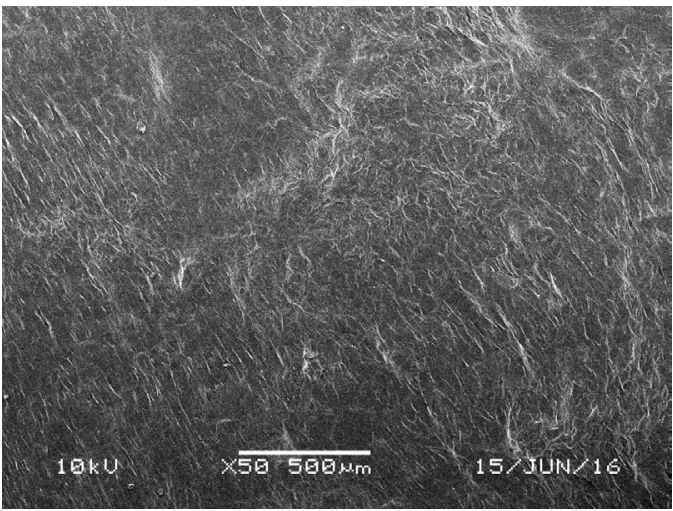

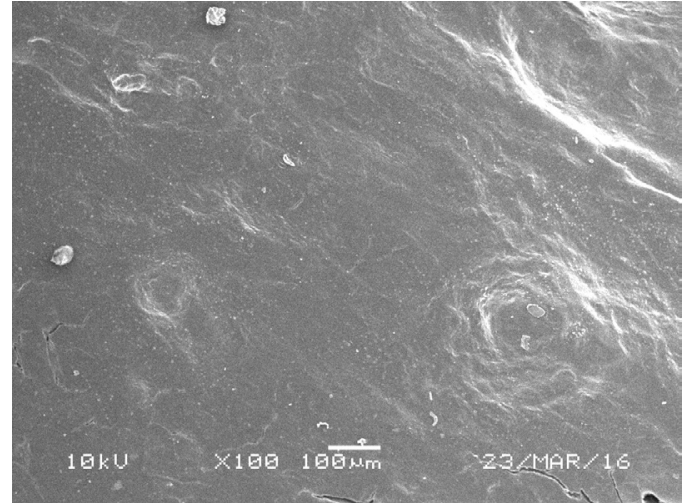
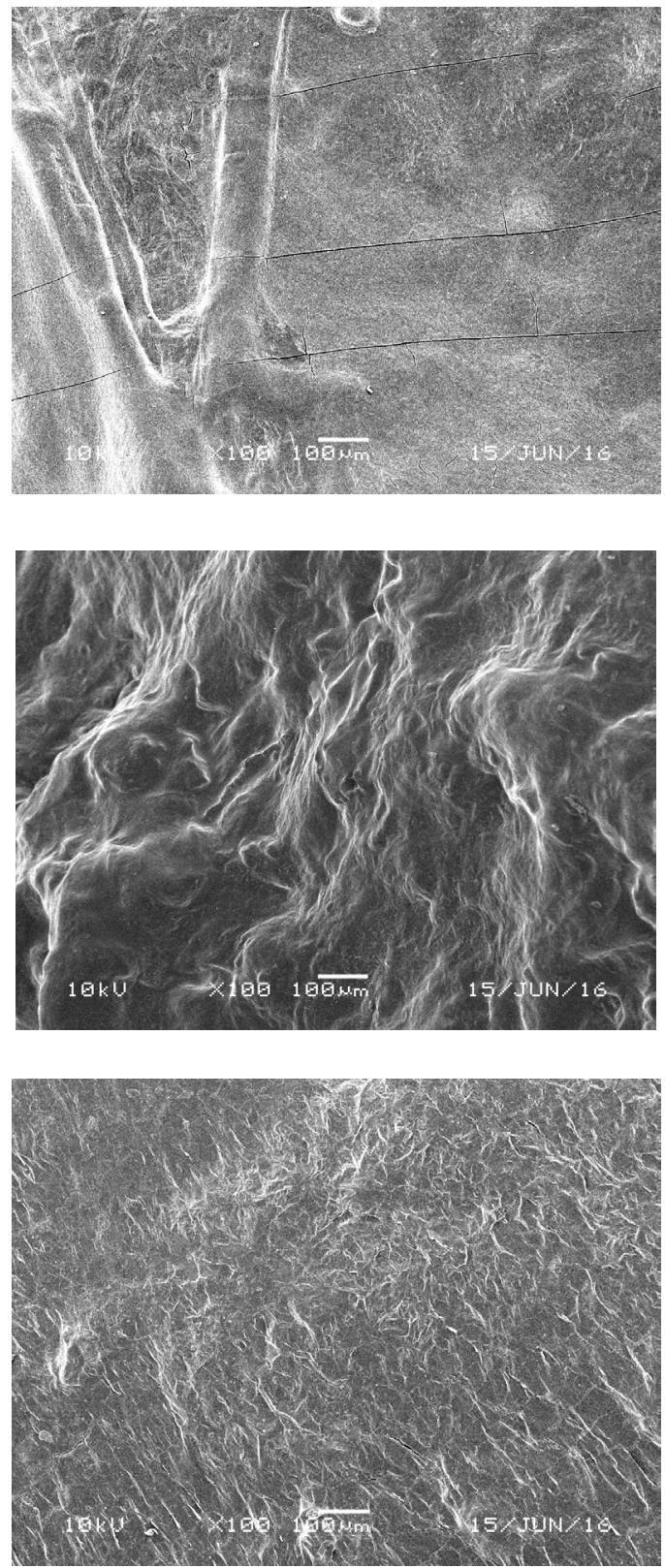

Figure 1. Illustration of the surface of placental tissues acquired using S.E.M. technique ( $x 35$ or $x 50 ; \times 100)$.

(A) healthy, appropriate-for-gestational-age (AGA) children born by healthy mothers; (B) children with intrauterine growth restriction (IUGR) born by healthy mothers; (C) children with intrauterine growth restriction (IUGR) born by mothers with pregnancy-induced hypertension; (D) large-for-gestational-age (LGA) children born by healthy mothers. 
Group A ( $n=8)$ : healthy mothers, routine and uneventful pregnancy, full-term-delivery neonates (bw 10th - 90th percentile);

Group B ( $n=3)$ : healthy mothers who delivered neonated with intrauterine growth restriction (IUGR) - (bw $<10$ th percentile);

Group C $(n=2)$ : mothers with pregnancy induced hypertension who delivered neonates with IUGR (bw $<10$ th percentile).

Group D ( $\mathrm{n}=3$ ): healthy mothers who delivered largefor-gestational-age neonates (LGA) (bw $>90$ th percentile).

Women eligible for the study underwent three ultrasound examinations. The first ultrasound test was performed between the 12th and 14th week of gestation, the second between the 20th and 22nd week and the third one between the 32nd and 33rd week. Neonates with IUGR were identified and defined during the third and final ultrasound test performed during pregnancy. Fetuses with in utero abdominal measurements below the 10th percentile of reference values were defined as fetuses with IUGR. IUGR was confirmed at birth if the neonatal weight was below the 10th percentile. All the fetuses had normal karyotypes and no malformations at birth.

Scanning Electron Microscopy. The placentas obtained during the delivery were immediately transported to the laboratory where a trophoblast specimens were extracted and subjected to microscopic examinations. A small amount of tissue was extracted from each sample and was shaken in the physiological saline solution for an hour. Rinsed tissue fragments were immersed in $60 \%$ ethanol for 15 minutes and dried in a laboratory oven at $35^{\circ} \mathrm{C}$. Imaging of the surface structure changes of physiological and pathological placenta tissue was conducted by Scanning Electron Microscopy using a JSM-5500LV scanning electron microscope supplied by JEOL. The samples were mounted on aluminum stubs and coated with gold (JFC 1200, JEOL). Detection of secondary (SE) and back-scattered electrons (BSE) was conducted with an accelerating voltage of $10 \mathrm{kV}$. Microphotographs were taken at magnifications $35 \times, 50 \times$ and $100 \times$.

The study was approved by the Bioethics Committee in Bielsko-Biała (No: 2016/02/11/4), which is in accordance with the Helsinki Declaration.

\section{RESULTS AND DISCUSSION}

The scanning electron microscope (S.E.M.), owing to a great depth of view, enables observation of the surface of solids without the need to apply complex preparatory techniques. Analysis of tissue surface by the SEM method enabled the creation of a database allowing assignation of placenta tissues to an adequate group and preliminary identification of pathology. Figure 1 presents images of the surface of placenta tissues selected out of four groups. The images were obtained by the scanning electron microscopy. Identification was performed on the basis of morphological features of the placenta tissues. The morphological status of the tissue was thoroughly assessed in each sample, taking into account the damage of cells on the surface. Samples from the group of healthy mothers who delivered healthy, full-term neonates (Group A) were characterized by a homogenous surface texture with natural corrugation. Samples from the group of healthy mothers who delivered neonates with IUGR (Group B) had areas with a homogenous surface texture and areas with noticeable heterogeneity of the surface. The surface in the samples from the group of mothers with pregnancy-induced hypertension who delivered neonates with IUGR (Group C) was definitely heterogeneous - noticeable swelling of the tissue surface was observed. Samples from the group of healthy mothers who delivered large-for-gestationalage neonates (Group D) also demonstrated a number of surface bulges and heterogeneities which were, nonetheless, characterized by a certain repeatability. Even with the low magnification of the microscope clear morphological differences in the examined placentas were observed, with the surface texture and the degree of tissue corrugation typical for a given group. The differences probably resulted from accumulation of various types of collagen fibers in the placenta. The highest heterogeneity occurred in the syncytiotrophoblast surface in mothers with pregnancy-induced hypertension who delivered neonates with IUGR, i.e. Group $\mathrm{C}$ - the group with the highest degree of pathology.

Structural disorders exposed in groups B, C and D reflect the disturbances in maternal-placental-fetal metabolism and, in particular, abnormalities in the process of intrauterine nutrition, in the transport of necessary components through the basal membrane and microvilli membrane of syncytiotrophoblast, as well as in the synthesis of various components, including hormones in syncytiotrophoblast. It is probable that in pathological placentas the accumulation of different types of collagen fibers occurs (Selim et al., 2013). The fibers interfere with the blood supply and lead to a gradual metabolic and transport dysfunction of the placenta. It cannot be ruled out that in pathologically altered placentas, like in preeclampsia, hypoxia of trophoblast cells occurs, leading to reduction of ATP biosynthesis and initiating oxidative stress and apoptosis of placenta cells (Bobiński et al., 2013b). The present pilot study demonstrates preliminary results of the analysis of placenta structure in the course of selected pathologies. In order to analyze the syncytiotrophoblast morphology in more detail, in the future the samples will be analyzed by transmission electron microscopy, immunohistochemical tests and FT RAMAN and FTIR spectroscopy analysis.

\section{REFERENCE}

Atwaa KA, Farghalyb LM (2011) Light and electron microscopic study of placental tissue in women suffering from severe preeclampsia. Egypt J Hist 34: 650-661. doi: 10.1097/01. EHX.0000406203.69444.59

Bobiński R, Mikulska M (2015) The ins and outs of maternal-fetal fatty acid metabolism. Acta Biochem Pol 62: 499-507. http://dx.doi. org/10.18388/abp.2015_1067

Bobiński R, Mikulska M, Mojska H, Simon M (2013a) Comparison of the fatty acid composition of maternal blood and cord blood of mothers who delivered healthy full-term babies, preterm babies, and full-term small for gestational age infants. I Maternal Fet Neonat Med 26: 96-102. doi: 10.3109/14767058.2012.722717

Bobiński R, Mikulska M, Mojska H, Simon M (2013b) Comparison of the fatty acid composition of transitional and mature milk of mothers who delivered healthy full-term babies, preterm babies, and fullterm small for gestational age infants. Europ J Clin Nutr 67: 966971. doi: $10.1038 /$ ejcn.2013.96

Duttaroy AK (2009) Transport of fatty acids across the human placenta: A review. Prog Lipid Res 48: 52-61. doi: 10.1016/j. plipres.2008.11.001

Elad D, Levkovitz R, Jaffa AJ, Desoye G, Hod M (2014) Have we neglected the role of fetal endothelium in transplacental transport? Traffic 15: 122-126. doi: 10.1111/tra.12130

Haggarty P (2002) Placental regulation of fatty acids delivery and its effect on fetal growth - a review. Placenta 16: 28-38.

Haggarty P (2004) Effect of placental function on fatty acid requirements during pregnancy. Eur J Clin Nutr 58: 1559-1570

Selim ME, Elshmry NG, Hamidi EI, Rashed A (2013) Electron and scanning microscopic observations on the syncytiotrophoblast microvillous membrane contribution to preeclampsia in early placental rats. J Blood Disorders Transf 4: 1-8. doi: 10.4172/2155-9864.1000137 